\title{
RNA-seq Analysis of Irrigated vs. Water Stressed Transcriptomes of Zea mays Cultivar Z59
}

\author{
B. Divya Bhanu ${ }^{1}$, Kandasamy Ulaganathan ${ }^{1 *}$, Arun K. Shanker ${ }^{2}$ and S. Desai ${ }^{2}$ \\ ${ }^{1}$ Centre for Plant Molecular Biology, Osmania University, Hyderabad, India, ${ }^{2}$ Central Research Institute for Dryland \\ Agriculture, Hyderabad, India
}

Keywords: RNA-seq, transcriptome, Zea mays, Z59, water stress, drought

\section{INTRODUCTION}

Maize (Zea mays) is one of the important food crops of India with other potential uses in preparation of livestock feed and biofuel. But production of maize in India is low compared to top maize producing countries due to abiotic stresses (Ranum et al., 2014). Among abiotic stresses affecting maize production in India, drought is the major limiting factor. In spite of extensive research on drought tolerance in maize no tangible achievements could be made due to the extreme complexity associated with drought tolerance (Ashraf, 2010). Availability

Edited by:

Marco Pellegrini,

Consiglio Nazionale delle Ricerche,

Italy

Reviewed by:

Romina D'Aurizio,

Consiglio Nazionale delle Ricerche,

Caroline Marcon,

University of Bonn, Germany

*Correspondence:

Kandasamy Ulaganathan

kulaganathan123@gmail.com

Specialty section:

This article was submitted to Bioinformatics and Computational

Biology,

a section of the journal

Frontiers in Plant Science

Received: 01 December 2015 Accepted: 12 February 2016

Published: 01 March 2016

Citation:

Divya Bhanu B, Ulaganathan K, Shanker AK and Desai S (2016)

RNA-seq Analysis of Irrigated vs.

Water Stressed Transcriptomes of Zea

mays Cultivar Z59.

Front. Plant Sci. 7:239.

doi: $10.3389 /$ fpls.2016.00239 of the maize genome and high throughput genomic methods have provided new tools for addressing the complexity associated with drought tolerance (Zivy et al., 2015). We have chosen the drought tolerant genotype Z59 and are developing transcriptomic resources for better understanding of drought tolerance of this cultivar. As part of this work, we have sequenced the irrigated and water stressed transcriptomes of Z59 cultivar and the dataset is reported here.

\section{MATERIALS AND METHODS}

\section{Plant Material Used}

Seedlings of Zea mays Z59 cultivar were grown in separate pots of uniform soil under greenhouse conditions. Plants were grown under well-watered conditions until they reached the early reproductive stage. Drought was imposed at early reproductive-phase by withholding irrigation for half of the plants. Both control plants (irrigated) and stressed plants (50\% relative water content) were used for transcriptome sequencing.

\section{Library Preparation and Transcriptome Sequencing}

Leaf tissue (lamina, topmost leaf) was collected from control- and drought-treated plants and stored in "RNAlater" solution (Thermo Fisher Scientific) at $-80^{\circ} \mathrm{C}$. RNA isolation was carried out using the RNeasy Plant kit (Quiagen, 2015). The leaf samples were ground to fine powder using liquid nitrogen in a mortar and pestle and subsequent isolation steps were as per the instructions provided in the RNeasy Plant Kit. The concentration and purity of the RNA extracted was evaluated using the NanoDrop Spectrophotometer (Thermo Scientific- 1000). The integrity of the extracted RNA was analyzed on the Bioanalyzer (Agilent, 2100). Irrigated and water stressed RNA samples with 6.9 and 7.8 RNA integrity numbers, respectively were used for library preparation.

Library preparation was performed using Illumina TruSeq RNA library protocol developed by Illumina Technologies (San Diego, CA). 1 ug of total RNA was subjected to PolyA purification of mRNA. Purified mRNA was fragmented for 8 minutes at elevated temperature $\left(94^{\circ} \mathrm{C}\right)$ in the 
TABLE 1 | Zea mays Z59 transcriptome statistics.

\begin{tabular}{lll}
\hline Feature & Irrigated & Water stressed \\
\hline NCBI project ID & PRJNA300830 & PRJNA300830 \\
NCBI biosample ID & SAMN04229490 & SAMN04229491 \\
NCBI SRA accession number & SRP065617 & SRP065617 \\
NCBI transcriptome & GECR00000000 & GECS00000000 \\
$\begin{array}{l}\text { accession number } \\
\text { Sequence type }\end{array}$ & Illumina Nextseq 500 & Illumina Nextseq 500 \\
Total number of reads & 34738228 & 43086142 \\
Read length & 76 & 76 \\
No. of De novo transcripts & 31399 & 30157 \\
\hline
\end{tabular}

presence of divalent cations and reverse transcribed with SuperScript III Reverse Transcriptase by priming with random hexamers. Second strand cDNA was synthesized in the presence of DNA polymerase I and RnaseH. The cDNA was cleaned up using HighPrep PCR (MAGBIO, Cat\# AC-60050). Illumina adapters were ligated to the cDNA molecules after end repair and addition of A base. SPRI (solid-phase reversible immobilization, Beckman Coulter) cleanup was performed after ligation. The library was amplified using 8 cycles of PCR for enrichment of adapter ligated fragments. The prepared library was quantified using Qubit and validated for quality by running an aliquot $(1 \mu \mathrm{l})$ on High Sensitivity DNA Kit (Agilent) which showed expected fragment distribution in the range of $\sim 250-500 \mathrm{bp}$. The effective sequencing insert size was $\sim 130-380 \mathrm{bp}$; the inserts were flanked by adapters whose combined size was $\sim 130 \mathrm{bp}$. Transcriptome sequencing was carried out with the Illumina-Nextseq500 system (Illumina, San Diego, CA).

\section{Preprocessing and Assembly of Transcripts}

The preprocessing of the reads was performed with FastQC (Andrews, 2010) and the adapters were removed by using Cutadapt tool (Martin, 2011). De novo transcriptome assembly was performed with Velvet and Oases software (Zerbino and Birney, 2008; Schulz et al., 2012).

\section{RESULTS}

Transcriptome sequencing resulted in 17369114 and 21543071 reads in irrigated and water stressed samples, respectively. The read length after trimming was found to be 76 base pairs. De novo assembly of the reads generated a total of 31399

\section{REFERENCES}

Andrews, S. (2010). FastQC: A Quality Control Tool for High Throughput Sequence Data. Available online at: http://www.bioinformatics.babraham.ac.uk/projects/ fastqc

Ashraf, M. (2010). Inducing drought tolerance in plants: recent advances. Biotechnol. Adv. 28, 169-183. doi: 10.1016/j.biotechadv.2009.11.005

Martin, M. (2011). Cutadapt removes adapter sequences from high-throughput sequencing reads. EMBnet. J. 17, 10-12. doi: 10.14806/ej.17.1.200 and 30157 transcripts in irrigated and water stressed samples of Z59, respectively. The summary of the transcriptomes are listed in Table 1. Further comparative analysis of irrigated and drought stressed transcriptomes is in progress to identify genes differentially expressed during drought stress and also to identify differential alternate splicing of transcripts during drought stress.

\section{Direct Link to Deposited Data and Information to Users}

The dataset submitted to NCBI include the assembled transcriptome sequences of irrigated and water stressed plants of Zea mays cultivar Z59 in Fasta format and the raw reads. The assembled transcriptome sequences of the irrigated and water stressed plants of Zea mays cultivar Z59 can be accessed at NCBI with the following accession numbers: GECR00000000 (irrigated); GECS00000000 (water stressed). Raw reads of both transcriptomes can be accessed with the following NCBI accession number: SRP065617. Users can download and use the data freely for research purpose only with acknowledgment to us and quoting this paper as reference to the data. Comparative analysis of Z59 (drought tolerant cultivar) transcriptomes with other transcriptomes made from other drought tolerant and susceptible maize cultivars can be used for effective identification of genes associated with drought tolerance in maize.

\section{AUTHOR CONTRIBUTIONS}

The work was planned jointly and executed by BD and KU. AS and SD were involved in selection and growth of the plant material.

\section{FUNDING}

The work was carried out with financial assistance from Department of Science and Technology, Ministry of Science and Technology, Government of India, New Delhi.

\section{ACKNOWLEDGMENTS}

We acknowledge the financial support to BD under the Women Scientist (A) scheme from the Department of Science and Technology, Ministry of Science and Technology, New Delhi. We acknowledge ICAR-CRIDA and National Innovations in Climate Resilient Agriculture (NICR) for seeds of drought tolerant genotype Z59.

Quiagen (2015). RNeasy Plant Kit. Available online at: https://www.qiagen.com/in/ shop/sample-technologies/rna/rna-preparation/rneasy-plant-mini-kit/

Ranum, P., Pena Rosas, J. P., and Garcia Casal, M. N. (2014). Global maize production, utilization, and consumption. Ann. N.Y. Acad. Sci. 1312, 105-112. doi: $10.1111 /$ nyas. 12396

Schulz, M. H., Zerbino, D. R., Vingron, M., and Birney, E. (2012). Oases: robust de novo RNA-seq assembly across the dynamic range of expression levels. Bioinformatics 28, 1086-1092. doi: 10.1093/bioinformatics/ bts094 
Zerbino, D. R., and Birney, E. (2008). Velvet: algorithms for de novo short read assembly using de Bruijn graphs. Genome Res. 18, 821-829. doi: 10.1101/gr.074492.107

Zivy, M., Wienkoop, S., Renaut, J., Pinheiro, C., Goulas, E., and Carpentier, S. (2015). The quest for tolerant varieties: the importance of integrating "omics" techniques to phenotyping. Front. Plant Sci. 6:448. doi: 10.3389/fpls.2015. 00448

Conflict of Interest Statement: The authors declare that the research was conducted in the absence of any commercial or financial relationships that could be construed as a potential conflict of interest.
The reviewer, RD, and handling Editor declared their shared affiliation, and the handling Editor states that the process nevertheless met the standards of a fair and objective review.

Copyright (๑ 2016 Divya Bhanu, Ulaganathan, Shanker and Desai. This is an openaccess article distributed under the terms of the Creative Commons Attribution License (CC BY). The use, distribution or reproduction in other forums is permitted, provided the original author(s) or licensor are credited and that the original publication in this journal is cited, in accordance with accepted academic practice. No use, distribution or reproduction is permitted which does not comply with these terms. 